\title{
Introduction to the Software Quality and Maintainability special issue
}

\author{
Yiannis Kanellopoulos $\cdot$ Rudolf Ferenc
}

Published online: 12 April 2012

(C) Springer Science+Business Media, LLC 2012

This special issue on Software Quality and Maintainability offers a venue to researchers to present their original work and to practitioners to relate their experiences on issues pertaining to software quality and maintainability. Moreover, the theme of this special issue opens discussion on how to bridge the gap between end user expectations, business requirements, vendor performance, and engineering constraints regarding software quality.

Out of 13 full-paper submissions for this issue, 6 papers were selected. The accepted papers address a range of topics related to software quality, including measurement, assessment, models, certificates, and tool support.

Various authors have discussed their ideas on the analysis of the quality of source code properties and its impact on a system's maintainability. Bijlsma et al. present an empirical study of the relation between technical quality of software products and the defect resolution performance of their maintainers.

Certification of software products and processes is reviewed in this special issue. Baggen et al. provide an approach in which, when a software system reaches a minimum level of maintainability, it can be certified with the TÜViT "Trusted Product Maintainability" certificate. Baldassare et al. discuss a specific process for comparing quality certifications, with specific reference to the CMMI-DEV and ISO 9001 models.

Quality analysis of complex source code bases requires sophisticated tool support. Kienle et al. are exploring an approach that utilizes system-specific static analyses of code with the goal to improve software quality for specific software systems, especially targeting complex embedded systems.

Franke et al. propose the use of a Bayesian decision support model, designed to help enterprise IT systems decision makers evaluate the consequences of their decisions by analyzing various scenarios. Lagerström et al. present a methodology that uses data from

Y. Kanellopoulos $(\square)$

Software Improvement Group, Amstelplein 1, 1096 HA Amsterdam, The Netherlands

e-mail: y.kanellopoulos@sig.eu

R. Ferenc

University of Szeged, Szeged, Pf. 652, 6701, Hungary

e-mail: ferenc@inf.u-szeged.hu 
50 projects performed at a large bank in Sweden to identify factors that have an impact on software development cost.

We are grateful to all the reviewers and to their sub-reviewers for helping to make this issue a success. 